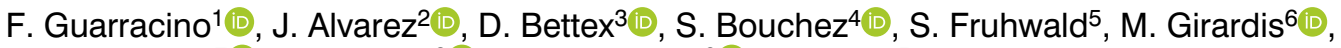

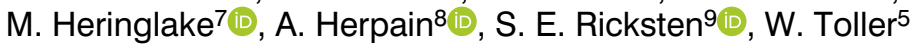

${ }^{1}$ Dipartimento di Anestesia e Terapie Intensive, Azienda Ospedaliero-Universitaria Pisana, Pisa, Italy

2Department of Anesthesia and Surgical ICU, University of Santiago de Compostela, Santiago de Compostela, Spain

${ }^{3}$ Cardiac Anaesthesia, University Hospital, Zürich, Switzerland

${ }^{4}$ Department of Anesthesiology, University Hospital, Ghent, Belgium

${ }^{5}$ Department of Anaesthesiology and Intensive Care Medicine, Division of Anaesthesiology for Cardiovascular Surgery and Intensive Care

Medicine, Medical University of Graz, Graz, Austria

${ }^{6}$ Struttura Complessa di Anestesia 1, Policlinico di Modena, Modena, Italy

${ }^{7}$ Klinik für Anästhesie und Intensivmedizin, Herz- und Diabeteszentrum Mecklenburg Vorpommern, Karlsburg, Germany

${ }^{8}$ Department of Intensive Care, Erasme University Hospital, Brussels, Belgium

${ }^{9}$ Department of Anesthesiology and Intensive Care, Sahlgrenska Universitetssjukhuset, Gothenburg, Sweden

\title{
Levosimendan comes of age: twenty years in clinical use
}

Key words: critical care, calcium sensitization, levosimendan, $\mathrm{K}_{\text {ATP }}$ channels, weaning, sepsis

Med Res J 2020; 5 (4): 219-221

\section{Background}

It is 20 years since the introduction of levosimendan in clinical use for short-term treatment of acutely decompensated heart failure - one of few novel medical treatments made available in recent times for the acute heart failure management. Levosimendan has been authorized for clinical use in over 60 countries and territories, and further activities are currently ongoing aimed at introducing it in China and the USA. In this issue of the Medical Research Journal, a review by Tritapepe et al. [1] systematically describes how this drug came to be and has been used in clinics in the last two decades. On our side, we wanted to highlight with this short editorial the most prominent features of levosimendan as a molecule and as a drug.

Levosimendan is an inodilator that augments cardiac contractility primarily via calcium sensitization of cardiac troponin $\mathrm{C}$ and mediates vascular dilatation via the opening of adenosine triphosphate-sensitive potassium $\left(\mathrm{K}_{\text {ATP }}\right)$ channels in vascular smooth muscle cells; the drug also exerts ischemia-protective action via a similar effect on mitochondrial $\mathrm{K}_{\text {ATP }}$ channels in several organs including the heart [2]. Of further note is that levosimendan promotes inotropy without increasing myocardial oxygen consumption. These aspects may be relevant in critical illness, when patients may be in a state of precarious energy balance.

\section{Acutely decompensated heart failure}

Levosimendan has been developed as a treatment for acutely decompensated heart failure. The efficacy and safety of levosimendan in its core indication have been established in randomized controlled trials involving over 9000 patients, supplemented by real-world experience. Comparison with other cardio- and vasoactive drugs meant for use in acute cardiac care shows superiority in hemodynamic, neurohormonal, and symptomatic effects without a sign of compromising long-term survival [3]. Since the beginning, however, its multifaceted pharmacology identified levosimendan as a drug that may have applications in a range of other critical illness situations. The conceptual framework for this wider use has been outlined in various recent critical care focused publications $[4,5]$.

\section{Perioperative hemodynamic support}

Despite a strong rationale and the comfort of many successful pilot studies [6] peri-operative use of levosimendan was not associated with a statistically significant impact on overall mortality in high-risk patients undergoing cardiac surgery in none of three recent trials [7]. However, a trajectory towards a survival benefit from levosimendan was noted in the LEVO-CTS trial, which 
also produced suggestions of greater clinical benefit in patients with severely reduced preoperative left ventricular ejection fraction (LVEF) $<35 \%$. In fact, pre-specified supplementary analyses confirmed a survival benefit in patients undergoing isolated coronary artery bypass grafting [8]. These tendencies have been corroborated in a meta-analysis that also identified a reduced risk of post-operative low cardiac output syndrome and reduced mechanical support requirements in patients with pre-operative LVEF $<35 \%(p=0.05)$ [4]. Additional notable findings included a lower overall incidence of acute kidney injury $(p=0.02)$ and less need for renal replacement therapy $(p=0.02)$. These latter findings speak to the proposition, addressed below, that specific renal actions of levosimendan-exercised at both glomerular and systemic hemodynamic levels - exert important renal-protective effects [9].

\section{In septic shock}

A plurality of theoretical considerations, experimental research and data from small clinical trials posit a beneficial impact of levosimendan in septic shock [5]. Against these must be set the findings of the LeoPARDS trial [10] that suggests that the use of levosimendan in septic shock patients may confer no overall clinical benefit in term of organ dysfunction prevention. In case of severe septic cardiomyopathy. Further research is needed to examine interplay with the degree of severity of cardiac failure plus the influence (if any) of treatment timing. In addition, the risk associated to the excessive use of vasoactive amines led to consider different approaches for the management of septic patients; in this context, levosimendan may represent a useful alternative to dobutamine in patients requiring inotropes, especially in case of concomitant ischemic cardiomyopathy.

\section{In weaning from respiratory and circulatory supports}

A substantial percentage of intubated patients in ICUs are difficult to wean from mechanical ventilation, with impacts on morbidity and mortality. Next to the problem of congestion in ventilatory weaning failure, is the development of diaphragm weakness. The pathophysiology of the latter process is complex but includes altered calcium sensitivity of the contractile proteins. Hence it is plausible that levosimendan may improve the prospects for successful weaning in some of these patients [11], although the only completed clinical study investigating this proposition has produced equivocal results and no indication of improved diaphragmatic neuromechanical efficiency but significant increases in both tidal volume and minute ventilation [12]. In the arena of weaning extracorporeal life support, there is evidence that levosimendan has some pertinent advantages over other inotropes, including no increase in myocardial oxygen consumption and an improvement in ventriculo-arterial coupling together with a prolonged cardiovascular effect and improvement in endothelial function [13].

\section{Renal function}

Exploratory clinical studies suggest a renal-protective effect of levosimendan in a range of cardiac low-output states that may be relevant to critical care or cardiorenal syndrome [8]. Pertinently, detailed clinical research has both clarified the effects of levosimendan on the glomerular vasculature and demonstrated that levosimendan-induced augmentation of glomerular filtration rate does not compromise renal oxygenation [14], thus differentiating levosimendan in functionally important ways from agents such as dobutamine [15] and milrinone [16].

\section{Conclusions}

Taken overall, the experience of the use of levosimendan in critical care settings is affirmative and the drug safety was proven even in such severe conditions. The effects of levosimendan differentiate in important ways from those of other cardio- and vasoactive drugs routinely used in the ICU. Nevertheless, it must be recognized that the size and power of some of the extant studies are limited and that many of the beguiling signals of clinical advantage that have been registered are in need of verification in larger, well-configured controlled trials. Experts' commentary on these outcomes nevertheless asserts the continuing potential of levosimendan [7]. The breadth of the exploratory applications in critical care scenarios may be regarded as a dividend of the original research program from which levosimendan emerged. An early commitment to detailed appreciation of this drug's pharmacology and effects on cellular processes has provided a basis from which the ingenuity of clinical researchers has been able to identify potential new applications. Levosimendan, therefore, enters its third decade in clinical service with much still to offer in a range of situations beyond acute heart failure.

Conflict of interest: $A$ ll authors received in the latest 5 years honoraria for educational lectures and/or grants for investigator initiated studies from Orion Pharma, where levosimendan has been discovered and developed. 


\section{References}

1. Tritapepe L, Pollesello P, Grossini E. The inodilator levosimendan: 20 years of experience in various settings of cardiac care. Med Res J. 2020; 5(4): 271-280, doi: 10.5603/MRJ.a2020.0037.

2. Papp Z, Édes I, Fruhwald S, et al. Levosimendan: molecular mechanisms and clinical implications: consensus of experts on the mechanisms of action of levosimendan. Int J Cardiol. 2012; 159(2): 82-87, doi: 10.1016/j.ijcard.2011.07.022, indexed in Pubmed: 21784540.

3. Li H, Duan Y, Chen B, et al. New pharmacological treatments for heart failure with reduced ejection fraction (HFrEF): A Bayesian network meta-analysis. Medicine (Baltimore). 2020; 99(5): e18341, doi: 10.1097/MD.0000000000018341, indexed in Pubmed: 32000355.

4. Sanfilippo F, Knight JB, Scolletta S, et al. Levosimendan for patients with severely reduced left ventricular systolic function and/or low cardiac output syndrome undergoing cardiac surgery: a systematic review and meta-analysis. Crit Care. 2017; 21(1): 252, doi: 10.1186/s13054017-1849-0, indexed in Pubmed: 29047417.

5. Herpain A, Bouchez S, Girardis M, et al. Use of Levosimendan in Intensive Care Unit Settings: An Opinion Paper. J Cardiovasc Pharmacol. 2019; 73(1): 3-14, doi: 10.1097/FJC.0000000000000636, indexed in Pubmed: 30489437.

6. Lim JuY, Deo SV, Rababa'h A, et al. Levosimendan Reduces Mortality in Adults with Left Ventricular Dysfunction Undergoing Cardiac Surgery: A Systematic Review and Meta-analysis. J Card Surg. 2015; 30(7): 547-554, doi: 10.1111/jocs.12562, indexed in Pubmed: 25989324.

7. Guarracino F, Heringlake M, Cholley B, et al. Use of Levosimendan in Cardiac Surgery: An Update After the LEVO-CTS, CHEETAH, and LICORN Trials in the Light of Clinical Practice. J Cardiovasc Pharmacol. 2018; 71(1): 1-9, doi: 10.1097/FJC.0000000000000551, indexed in Pubmed: 29076887.

8. van Diepen S, Mehta RH, Leimberger JD, et al. Levosimendan in patients with reduced left ventricular function undergoing isolated coronary or valve surgery. J Thorac Cardiovasc Surg. 2020; 159(6): 2302-2309.e6, doi: 10.1016/j.jtcvs.2019.06.020, indexed in Pubmed: 31358329 .

9. Yilmaz MB, Grossini E, Silva Cardoso JC, et al. Renal effects of levosimendan: a consensus report. Cardiovasc Drugs Ther. 2013: 27(6): 581590, doi: 10.1007/s10557-013-6485-6, indexed in Pubmed: 23929366

10. Gordon A, Santhakumaran S, Al-Beidh F, et al. Levosimendan to prevent acute organ dysfunction in sepsis: the LeoPARDS RCT. Efficacy and Mechanism Evaluation. 2018: 5(6): 1-94 doi: 10.3310/eme05060.

11. Sangalli $F$, Bellani $G$, Affronti $A$, et al. Levosimendan to facilitate weaning from cardiorespiratory support in critically ill patients: current evidence and future directions. Minerva Anestesiol. 2020; 86(6): 645-651, doi: 10.23736/S0375-9393.20.14219-6, indexed in Pubmed: 32013333.

12. Roesthuis $L$, van der Hoeven $H$, Sinderby $C$, et al. Effects of levosimendan on respiratory muscle function in patients weaning from mechanical ventilation. Intensive Care Med. 2019; 45(10): 1372-1381, doi: 10.1007/s00134-019-05767-y, indexed in Pubmed: 31576436.

13. Distelmaier $K$, Roth $C$, Schrutka $L$, et al. Beneficial effects of levosimendan on survival in patients undergoing extracorporeal membrane oxygenation after cardiovascular surgery. Br J Anaesth. 2016; 117(1): 52-58, doi: 10.1093/bja/aew151, indexed in Pubmed: 27317704.

14. Bragadottir G, Redfors B, Ricksten SE. Effects of levosimendan on glomerular filtration rate, renal blood flow, and renal oxygenation after cardiac surgery with cardiopulmonary bypass: a randomized placebo-controlled study. Crit Care Med. 2013; 41(10): 2328-2335, doi: 10.1097/CCM.0b013e31828e946a, indexed in Pubmed: 23921271.

15. Lannemyr L, Ricksten SE, Rundqvist B, et al. Differential Effects of Levosimendan and Dobutamine on Glomerular Filtration Rate in $\mathrm{Pa}$ tients With Heart Failure and Renal Impairment:A Randomized DoubleBlind Controlled Trial. J Am Heart Assoc. 2018; 7(16): e008455, doi: 10.1161/JAHA.117.008455, indexed in Pubmed: 30369310.

16. Lannemyr L, Bragadottir $G$, Redfors $B$, et al. Effects of milrinone on renal perfusion, filtration and oxygenation in patients with acute heart failure and low cardiac output early after cardiac surgery. J Crit Care. 2020; 57: 225-230, doi: 10.1016/j.jcrc.2019.12.022, indexed in Pubmed: 31919012. 